\title{
Characterization of graphene-based photonic crystal in THz spectrum with finite-difference time domain method*
}

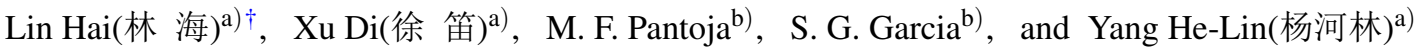 \\ a) College of Physical Science and Technology, Central China Normal University, Wuhan 430079, China \\ b) Department of Electromagnetic and Physics Matter, University of Granada, Spain
}

(Received 16 January 2014; revised manuscript received 26 March 2014; published online 16 July 2014)

\begin{abstract}
Graphene has been considered as a promising material which may find applications in the $\mathrm{THz}$ science. In this work, we numerically investigate tunable photonic crystals in the THz range based on stacked graphene/dielelctric layers, a complex pole-residue pair model is used to find the effective permittivity of graphene, which could be easily incorporated into the finite-difference time domain (FDTD) algorithm. Two different schemes of photonic crystal used for extending the bandgap have been simulated through this FDTD technique.
\end{abstract}

Keywords: graphene, photonic cyrstal

PACS: 42.25.Bs, 81.05.ue

DOI: $10.1088 / 1674-1056 / 23 / 9 / 094203$

\section{Introduction}

Graphene has attracted a great deal of interest in the field of electronics and photonics. ${ }^{[1,2]}$ As a two-dimensional monoatomic layer of carbon arranged in a honey-comb lattice, it has remarkable flexibility, robustness, and environmental stability, as well as extraordinary optical properties. ${ }^{[3]}$ Numerous graphene devices including field-effect transistors, multipliers, modulators, and surface plasmon waveguiding devices have been proposed and/or envisaged. ${ }^{[4,5]}$ However the application of graphene in photonic crystal devices operating in the far-infrared to near-infrared spectral range has been far less exploited. This is mainly due to the limitations in fabricating and transfer technique for doped graphene. Recently, in Ref. [6], through multiple layer deposition steps and a single lithography step, researchers fabricated a patterned plasmonic device consisting of stacks of graphene/insulator disks. Through this technique, it is now possible to fabricate one-dimensional artificially periodic media which are composed of periodically arranged graphene and insulator.

Meanwhile, the optical transmission through the above periodical graphene/insulator structure has become a hot topic and has attracted some interest of the nanophotonic community. Very recently, Kaipa et al. ${ }^{[7]}$ reported their finding on the transmissivity of a low-terahertz electromagnetic wave through a stack of monolayer graphene sheets separated by dielectric slabs. Some other researchers ${ }^{[8,9]}$ reported the achievement of photonic crystals (PCs) in the far-infrared region through adding graphene on the top of dielectric photonic crystals.

The enhanced widths as well as highly tunable bandgaps in PCs could be of great interest to the THz community. Researchers hope to take advantage of graphene's tunability to design highly tunable THz PCs or metamaterials. ${ }^{[10]}$ Herein, we present our recent exploration of a graphene-dielectric periodic stack. In the proposed periodical multilayer structure, a one-atom thick graphene layer is added to the original all-dielectric photonic crystal, i.e., each period is made of graphene and two different types of insulators. With a suitable gate voltage, the real part of the equivalent relative permittivity of the graphene sheet can be tuned, which can provide tunability to the bandgap width of the PC. The numerical simulation results are obtained through full-wave finite-difference time domain (FDTD) simulation. In the Yee grids, the graphene sheets are treated as a dispersive thin sheet, a complex conjugate pole dispersive model is used to describe the effective permittivity in the frequency domain, which can be easily transformed into FDTD updating schemes. To validate our numerical simulation, we first compare our FDTD results with the transfer-matrix method (TMM) results in Ref. [7]. Then, two different schemes used for extending the photonic bandgap (PBG) of the graphene PC are simulated using the same technique.

\section{Graphene model}

The unique light-matter interaction properties of graphene originate from its high surface conductivity $\sigma_{2 \mathrm{D}}$, which could be tuned using the surrounding material. This important feature enables wide possibilities in realizing tunable optoelectronic devices and metamaterials based on graphene. An applied external electric field produces changes in the

* Project supported by the Spanish Ministry of Education (Grant No. SB2010-0056), the EU FP7/2007-2013 (Grant No. 205294) (HIRF SE project), the Spanish National Projects (Grant Nos. TEC2010-20841-C04-04 and CSD2008-00068), the Junta de Andalusia Project (Grant No. P09-TIC-5327), the National Natural Science Foundation of China (Grant No. 60906030), and the Self Research Program of Central China Normal University (Grant No. 09010028).

†Corresponding author. E-mail: linhai.ccnu@gmail.com

(C) 2014 Chinese Physical Society and IOP Publishing Ltd

http://iopscience.iop.org/cpb http://cpb.iphy.ac.cn 
chemical potential $\mu_{\mathrm{c}}$ and in the surface conductivity $\sigma_{2 \mathrm{D}}$ of graphene, which can be expressed as ${ }^{[11]}$

$$
\begin{aligned}
\sigma_{2 \mathrm{D}}(\omega)= & \mathrm{i} \frac{1}{\pi \hbar^{2}} \frac{e^{2} k_{\mathrm{B}} T}{\omega-\mathrm{i} 2 \Gamma}\left\{\frac{\mu_{\mathrm{c}}}{k_{\mathrm{B}} T}+2 \ln \left[\exp \left(-\frac{\mu_{\mathrm{c}}}{k_{\mathrm{B}} T}\right)+1\right]\right\} \\
& +\mathrm{i} \frac{e^{2}}{4 \pi \hbar} \ln \left[\frac{2\left|\mu_{\mathrm{c}}\right|-\hbar(\omega-\mathrm{i} 2 \Gamma)}{2\left|\mu_{\mathrm{c}}\right|+\hbar(\omega-\mathrm{i} 2 \Gamma)}\right]
\end{aligned}
$$

where $\omega$ is the angular frequency, $\Gamma$ is the scattering rate, $T$ is the absolute temperature, $-e$ is the charge of an electron, $\hbar=h / 2 \pi$ is the reduced Planck's constant, and $k_{\mathrm{B}}$ is the Boltzmann's constant.

\section{Numerical algorithm}

In order to investigate the EM response of graphene and the surrounding environments through computation electromagnetic methods, an adequate method is to find a proper representation of graphene as a thin three-dimensional layer. To this end, it is convenient to formulate the conductivity as a contribution to the equivalent permittivity

$$
\tilde{\varepsilon}(r, \omega)=\varepsilon(r, \omega)-\mathrm{i} \frac{\sigma(r, \omega)}{\omega} .
$$

For graphene, we only need to divide the two-dimensional conductivity with an assumed thickness which is very small when compared to the minimum wavelength of interest, thus obtaining a three-dimensional analog conductivity. Then, the effective permittivity of graphene can be obtained as

$$
\tilde{\varepsilon}(\omega)=\varepsilon_{0} \varepsilon_{\mathrm{m}}(\omega)=\varepsilon_{0}\left(1-\mathrm{i} \frac{\sigma_{2 \mathrm{D}}(\omega)}{\varepsilon_{0} \omega d}\right) .
$$

Therefore, the graphene can be numerically considered as an isotropic frequency-dependent material like noble metals in plasmonics. Several numerical techniques have been proposed to study the interaction/propagation of electromagnetic waves with graphene, one of the most popular and widely accepted techniques is the FDTD, since the frequency response of the system under study over a wide range of frequencies can be obtained with a single run of simulation.

In order to obtain the wide spectrum response of a dispersive material, a proper frequency-domain model is highly desirable. The traditional Drude-Lorentz (DL) model or the Drude-critical point (DCP) model has been widely used, which can represent well the optical properties of the metal originated from the inter-band and intra-band transitions. For the case of graphene, while the intra-band relaxation contribution to graphene's permittivity can be represented using the Drude model alone, the inter-band transition contribution cannot be incorporated into the FDTD algorithm directly. To provide a numerically stable updating scheme in the FDTD, a complex conjugate model is used to represent the permittivity of graphene in the FDTD. Through this complex conjugate model, the permittivity of graphene can be expressed as

$$
\varepsilon_{\mathrm{eq}}=\varepsilon_{0} \varepsilon_{\infty}+\varepsilon_{0} \sum_{p}\left(\frac{c_{p}}{\mathrm{j} \omega-a_{p}}+\frac{c_{p}^{*}}{\mathrm{j} \omega+a_{p}^{*}}\right),
$$

where $c_{p}^{*}$ is the conjugate of $c_{p}$. By means of introducing complex poles and residues, a vector fitting modeling process can be utilized to find the poles and residues of this model. The sample value of graphene's permittivity is used as the input of the vector fitting toolbox. An ADE technique can be applied to implement the complex-conjugate dispersive material model in the FDTD. Following Refs. [12] and [13], the FDTD updating equation for the $E$ field is

$$
=\boldsymbol{E}^{(n)}+\frac{2 t \cdot\left[\boldsymbol{\nabla} \times \boldsymbol{H}^{(n+1 / 2) t}-\operatorname{Re} \sum_{p=1}^{p}\left(1+k_{p}\right) \boldsymbol{J}_{p}^{n t}\right]}{2 \varepsilon_{0} \varepsilon_{\infty}+\sum_{p=1}^{p} 2 \operatorname{Re}\left(\beta_{p}\right)},
$$

where $J_{p}$ is the auxiliary current introduced by the complexconjugate pole-residue pair. The magnetic field update equation remains unchanged. The auxiliary currents are derived as

$$
\boldsymbol{J}_{p}^{(n+1) t}=k_{p} \boldsymbol{J}_{p}^{n t}+\beta_{p}\left(\frac{\boldsymbol{E}^{(n+1)}-\boldsymbol{E}^{n t}}{t}\right),
$$

where coefficients $k_{p}$ and $\beta_{p}$ can be calculated from the given poles and residues as

$$
\begin{aligned}
& k_{p}=\frac{1+a_{p} t / 2}{1-a_{p} t / 2}, \\
& \beta_{p}=\frac{\varepsilon_{0} c_{p} t}{1-a_{p} t / 2} .
\end{aligned}
$$

\section{Structure of graphene PCs}

In recent years, researchers have tended to investigate PCs with wide and tunable photonic bandgaps. ${ }^{[14]}$ The tunability can be obtained by incorporating liquid crystals, semiconductors, or ferroelectric materials into the unit cells of PCs. According to the tunable nature of graphene, it is straightforward to achieve a tunable PC by integrating a graphene layer into the period of a dielectric PC, as illustrated in Fig. 1.

An enhanced PBG can be successfully achieved by means of mixing a ternary photonic crystal with a heterostructured photonic crystal. In this work, we would like to analyze the results of this method in the far-infrared regime. Two schemes to enhance the bandgap were proposed in Refs. [15] and [16]. In scheme 1, the graphene layer is added to every unit cell of the original binary dielectric photonic crystal so that the structure becomes ternary, i.e., each period is composed of graphene and two different types of dielectrics. The second scheme is to use the photonic heterostructure, which is formed by adding two or more ternary graphene/insulator PCs with different filling factors. The two schemes are illustrated in the Fig. 1. 


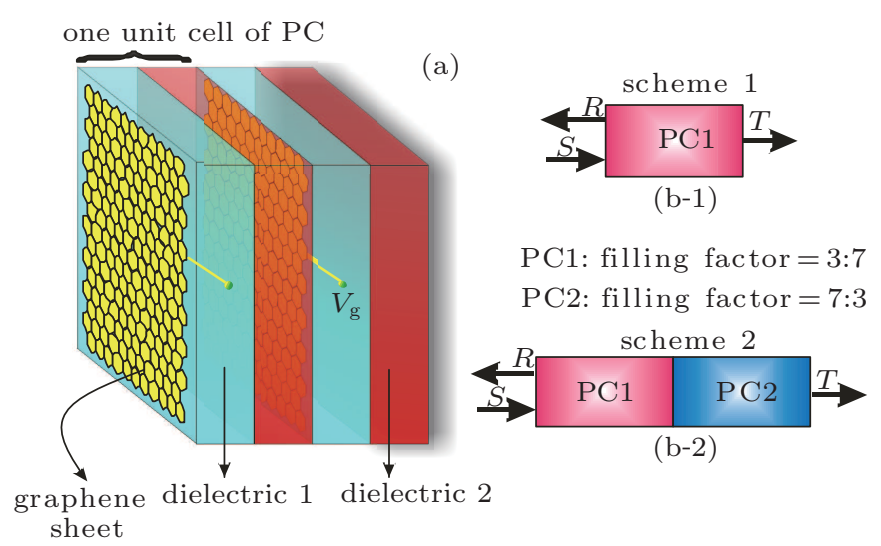

Fig. 1. (color online) (a) Unit cell of the graphene-based PC as well as (b) two schemes proposed to obtain an enhanced bandgap.

\section{Numerical validation}

First, we validate the accuracy and the effectiveness of our FDTD codes with the results presented in Ref. [7], which were obtained through the transfer-matrix method and the high-frequency structure simulator (HFSS) simulation. The structure under investigation with a plane wave at normal incidence is composed of four independently standing graphene sheets separated by dielectric slabs.

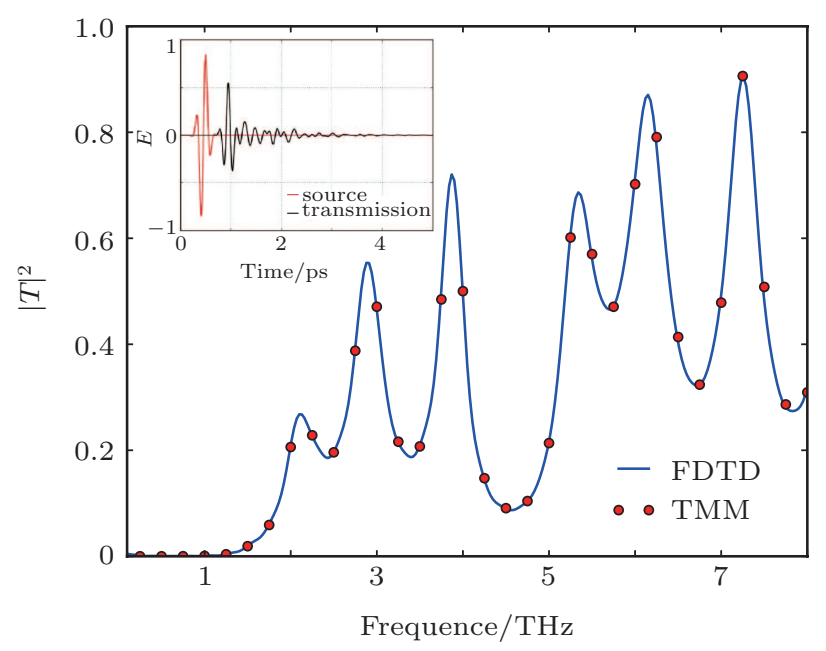

Fig. 2. (color online) Comparison between the FDTD calculated result and the transfer-matrix result.

The relative permittivity of the dielectric slab is 10.2 , and its height is $10 \mu \mathrm{m}$. Due to the symmetry of the problem, it can be solved through the one-dimensional FDTD simulation. The grid size is set to $10 \mathrm{~nm}$ and the time step is set to $0.006 \mathrm{fs}$. Since the thickness of the graphene is set to $1 \mathrm{~nm}$, which is only one-tenth of the grid size, an averaged procedure is used to achieve a better accuracy. By using the above described method, the wideband transmittivity response of the proposed structure can be obtained through the fast Fourier transform (FFT) of the time domain signals. A comparison between the FDTD calculated results and the transfer-matrix result in Ref. [7] is shown in Fig. 2. The agreement is quite good with a slight error, which may be caused by the inaccuracy of the FFT and dispersion.

Next we consider the proposed tunable structure based on graphene in the far-infrared regime. The structure is composed of ten unit cells, each being made up of one layer of graphene and two different types of dielectrics, as depicted in scheme 1 . The relative permittivity of dielectric 1 (D1) is 3.9 , while that of dielectric 2 (D2) is 11.7. Their thicknesses are $3 \mu \mathrm{m}$ and $7 \mu \mathrm{m}$, respectively. Different chemical potentials $\mu_{\mathrm{c}}$ are set to the doped graphene sheets.

Table 1. Fitted pole-residue pairs for $T=300 \mathrm{~K}, \tau=1.32 \mathrm{meV}$. Here $\varepsilon_{\infty}=2.279826567473442\left(\mu_{\mathrm{c}}=0.5\right), \varepsilon_{\infty}=7.440925961021825$ $\left(\mu_{\mathrm{c}}=0.1\right)$, and $\varepsilon_{\infty}=1.638285787916163\left(\mu_{\mathrm{c}}=1\right)$.

\begin{tabular}{cccc}
\hline$\mu_{\mathrm{c}}$ & $p$ & $a_{p}(\cdot 1015)$ & $c_{p}(\cdot 1018)$ \\
\hline & 1 & 0 & 0.335071 \\
0.1 & 2 & -0.004011 & -0.335013 \\
& 3 & $-0.004022+0.391271 \mathrm{i}$ & $-0.000016-0.001557 \mathrm{i}$ \\
& 4 & $-0.004022-0.391271 \mathrm{i}$ & $-0.000016+0.001557 \mathrm{i}$ \\
\hline \multirow{4}{*}{0.5} & 1 & 0 & 1.657353 \\
& 2 & -0.004011 & -1.657341 \\
& 3 & $-0.004011+1.961313 \mathrm{i}$ & $-0.000003-0.001569 \mathrm{i}$ \\
& 4 & $-0.004011-1.961313 \mathrm{i}$ & $-0.000003+0.001569 \mathrm{i}$ \\
\hline & 1 & 0 & 3.314689 \\
& 2 & -0.004011 & -3.314684 \\
& 3 & $-0.004131+3.926890 \mathrm{i}$ & $-0.000002-0.001574 \mathrm{i}$ \\
& 4 & $-0.004131-3.926890 \mathrm{i}$ & $-0.000002+0.001574 \mathrm{i}$ \\
\hline
\end{tabular}

The fitted $a_{p}$ and $c_{p}$ for this case are listed in Table 1. The considered frequency ranges from $0.1 \mathrm{THz}$ to $9 \mathrm{THz}$, which covers the entire spectrum of interest. The transmission coefficients under different $\mu_{\mathrm{c}}$ are depicted in Fig. 3. It can be observed that there is no significant change in the frequency of the left band edge for $\mu_{\mathrm{c}}=0.1 \mathrm{eV}, 0.5 \mathrm{eV}, 1 \mathrm{eV}$. However, the right band edge of the ternary $\mathrm{PC}$ moves to a higher frequency. As a result, the PBG is tuned due to the presence of graphene sheets and the changes of its chemical potential.

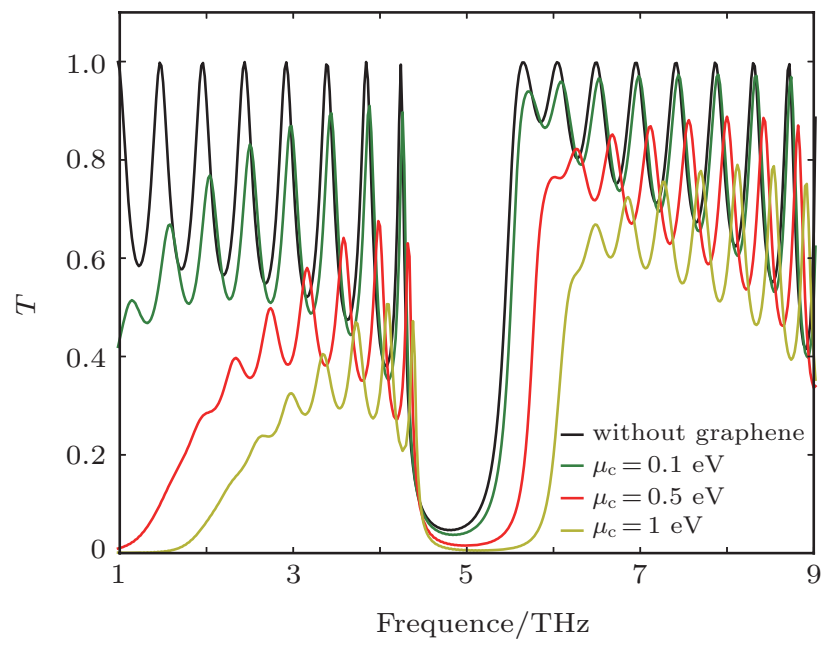

Fig. 3. (color online) Transmission coefficients of the ternary structure with different $\mu_{\mathrm{c}}$ and without graphene.

We further investigate the photonic bandgap of the graphene/insulator ternary PC with different filling factors, 
then mix two PCs having different filling factors together as the proposed scheme 2. The size of the PC's unit cell remains unchanged at $10 \mu \mathrm{m}$. The simulation results are presented in Fig. 4. It can be seen that the PBG moves to a higher frequency with the increase of the filling factor (D1:D2).

For the case of scheme 2, a 10-unit-cell arrangement of PC1 with a filling factor of 3:7 and a 10-unit-cell arrangement of PC2 with a filling factor of 7:3 are combined together. We can see that the employment of the graphene sheets increases the gap width by only about $0.5 \mathrm{THz}$ (under $\mu_{\mathrm{c}}=0.5 \mathrm{eV}$ ) in Fig. 4. The chemical potential $\mu_{\mathrm{c}}$ is tuned from $0.5 \mathrm{eV}$ to $1 \mathrm{eV}$ for this hetero-structure based on graphene. Through comparing the results, we can observe that the right band edge of the heterostructured $\mathrm{PC}(\mathrm{PC} 1+\mathrm{PC} 2)$ is slightly shifted to a higher frequency with the increase of $\mu_{\mathrm{c}}$.

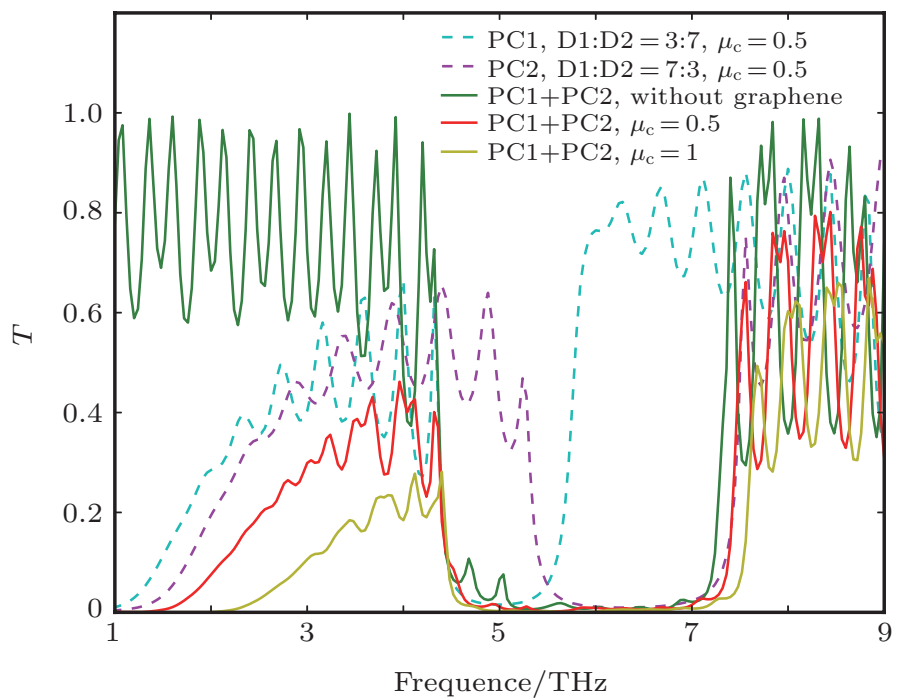

Fig. 4. (color online) Transmission coefficients of scheme 1 and scheme 2 with different filling factors

\section{Conclusion}

From the numerical simulation results, it is noticed that in the PC structure, the status of graphene is similar to that in a noble metal as shown in Ref. [15]. The difference is that graphene not only provides a bandgap extension to the original dielectric photonic crystal but also provides tunability to the bandgap. The limitation of the proposed graphene PC is its tuning ability. The tunability restriction may be overcome by patterning the doped graphene sheet to achieve a graphenebased metamaterial. ${ }^{[8]}$ Therefore, our efforts can be viewed as a first step towards the characterization of the 1D graphenebased tunable graphene metamaterial using the FDTD algorithm.

\section{References}

[1] Bao Q L and Kian P L 2012 ACS Nano 63677

[2] Wu H Q 2013 Chin. Phys. B 22098106.

[3] Stauber T, Peres N and Geim A 2008 Phys. Rev. B 78085432

[4] Grigorenko A, Polini M and Novoselov K 2012 Nature 6749

[5] Wu B 2014 Scientific Reports 4525

[6] Yan H, Li X, Chandra B, Tulevski G, Wu H and Freitag M 2012 Nat. Nanotechnol. 7330

[7] Kaipa C S R, Yakovlev A B, Hanson G W, Padooru Y R, Medina F and Mesa F 2012 Phys. Rev. B 85245407

[8] Sreekanth K V, Zeng S, Shang J, Yong K T and Yu T 2012 Sci. Rep. 2 737

[9] Berman O L and Kezerashvili R Y J 2012 Phys. Condens. Matter 24 015305

[10] Ju L 2011 Nat. Nanotech. 6630

[11] Hanson G W 2008 Dyadic Journal of Applied Physics 103064302

[12] Han M, Dutton R and Fan S 2006 IEEE Microw. Wireless Comp. Lett. 16119

[13] Lin H, Pantoja M F, Angulo L D, Alvarez J, Martin R G and Garcia S G 2012 IEEE Microw. Wireless Comp. Lett. 22612

[14] Lei J 2013 Chin. Phys. B 22104101

[15] Hung H C, Wu C J, Yang T J and Chang S J 2012 Progress in Electromagnetics Research 125219

[16] Wu C J, Hsieh Y C and Hsu H T 2011 Progress in Electromagnetics Research 114271 